\title{
Zum Stellenwert des Deutschen in Polen
}

\section{The status of the German language in Poland}

\begin{abstract}
The German language in Poland has a very long tradition dating back to the 18th century. Over the centuries, under the influence of historical, political, economic and social factors, the image of Germany and Germans in Poland as well as the attitude of Poles towards the German language have been subject to continual evolution. The aim of this article is to discuss the position of the German language in Poland, mainly in the $21^{\text {st }}$ century. Particular attention is paid to the discussion of the importance of this language in the Polish educational system in the past two decades, and the changing number of people learning, predominantly in primary and secondary education. Moreover, the reasons for the relatively high level of dislike of the German language among Poles (including learners of German) are presented and analysed. The discussion of the current status of German in our country is preceded by a brief historical outline showing the significance of the German language in Poland as well as the attitude of Poles towards this language over the centuries, beginning with the Old-Polish period.
\end{abstract}

KEYWORDS: German language in Poland, image of Germany and Germans in Poland, attitude of Poles towards the German language, German as a foreign language in primary and secondary education in Poland.

SCHLÜSSELWÖRTER: deutsche Sprache in Polen, Deutschlandbild in Polen, Einstellung der Polen dem Deutschen gegenüber, Deutsch als Fremdsprache in der Primar- und Sekundarschulbildung in Polen.

\section{EINLEITENDE BEMERKUNGEN}

Die Autoren zahlreicher Veröffentlichungen, die der Situation der deutschen Sprache u. a. in Polen ${ }^{1}$ gewidmet sind, berufen sich oft auf den Bericht des Auswärtigen Amtes Deutsch als Fremdsprache weltweit. Datenerhebung $2015^{2}$. Laut diesem Dokument lernten im Jahr 2015 15,5 Millionen Menschen Deutsch. An der Spitze der Länder, in denen Deutsch gelernt wurde, befand sich Polen mit 2,3 Millionen Deutschlernenden, davon 2,1 Millionen an Schulen, in denen

\footnotetext{
${ }^{1}$ Siehe u a. Földes (2016), Janoszczyk (2016), Skowronek (2016), Szczęk und Kałasznik (2016), Łada (2017).

${ }^{2}$ http:/ / www.auswaertiges-amt.de/cae/servlet/contentblob/364458/publicationFile/204449/PublStatistik.pdf [Zugriff am: 15.04.2019].
} 
seit Jahren Deutsch als zweite am häufigsten unterrichtete Fremdsprache (nach Englisch) gilt. Die aufgeführten Zahlen scheinen sehr optimistisch.

Dieser Beitrag setzt sich zum Ziel den Stellenwert der deutschen Sprache in Polen besonders im 21. Jahrhundert zu untersuchen. Der Beleuchtung des aktuellen Ranges des Deutschen in Polen geht ein historischer Überblick voraus, der die Position der deutschen Sprache als Verkehrsmittel und Unterrichtsgegenstand in Polen im Laufe der Jahrhunderte aufzeigt. Im abschließenden Teil dieses Beitrags werden die Gründe für die relativ hohe emotionelle Abneigung gegen die deutsche Sprache in Polen aufgeführt sowie einige Empfehlungen formuliert, deren Berücksichtigung zur Steigerung der Motivation zum Deutschlernen bei den polnischen SchülerInnen beitragen könnte. Um die aktuelle Bedeutung der deutschen Sprache in Polen sowie heutige Einstellung dem Deutschen selbst und seinen Sprechern gegenüber zu bestimmen, ist es notwendig, sich mit der Geschichte der deutsch-polnischen Beziehungen auseinanderzusetzen.

\section{DIE STELLUNG DER DEUTSCHEN SPRACHE IN POLEN - HISTORISCHER UMRISS}

Die Ausführungen über die deutsch-polnischen Sprachkontakte sollen ihren Anfang in der Erklärung des polnischen Wortes Niemcy (die Deutschen) nehmen, das etymologisch gesehen soviel wie die Stummen bedeutete. Da es unmöglich zu sein scheint, dass unsere polnischen Vorfahren ihre westlichen anderssprechenden Nachbarn als stumm wahrnahmen, ist diese Bezeichnung eher symbolisch $\mathrm{zu}$ verstehen. Vermutlich wurde damit eine Menschengruppe gemeint, mit denen die Polen keine sprachlichen Kontakte in einer der slawischen Sprachen ${ }^{3}$ aufnehmen konnten. ${ }^{4}$ Die zweite, und wahrscheinlichere, Deutung des Wortes geht auf den von Tacitus erwähnten Namen eines der germanischen Stämme, nämlich Nemetes, zurück (vgl. Kopaliński 2007: 343).

In der altpolnischen Zeit ${ }^{5}$ nahmen die Kontakte der Polen zu den Deutschen verschiedene Formen an. Auf eine interessante Art und Weise wird das altpolnische Bild des Deutschen von Bystron (1935/1995: 170, 178-179; siehe dazu auch Mihułka 2016b) dargestellt, der die Sichtweise des polnischen Adels und des

${ }^{3}$ Niemiec (der Deutsche) bildet die Opposition zu Stowianin (dem Slawen), dessen Name wiederum von stowo (Wort) abgeleitet wird und einen Menschen bezeichnet, mit dem man sich problemlos verständigen kann (vgl. Brzozowska 2008: 120-121).

${ }^{4}$ Mit Niemiec (der Deutsche) bezeichneten die Polen nicht nur ihre westlichen Nachbarn, sondern manchmal Fremde im Allgemeinen (vgl. Brzozowska 2008: 121).

${ }^{5}$ Die altpolnische Periode umfasst die Zeitspanne vom Jahr 1136 bis zur Wende des 15. Jahrhunderts. 
einfachen Volkes berücksichtigte und auf zwei Modelle des Deutschen verwies, die zugleich den Grad des gegenseitigen In-Kontakt-Tretens widerspiegelten. Das einfache Volk sah in dem Deutschen einen Fremden, der nicht "normal“ sprechen und mit dem man sich in keiner „normalen“, d. h. slawischen Sprache, verständigen konnte. Das Deutsche wurde als eine schwierige, unverständliche Sprache betrachtet, die oft mit der Bezeichnung szwargot ${ }^{6}$ versehen wurde (vgl. Bystroń 1935/1995: 170, 178-179). Im Gegensatz zum polnischen einfachen Volk, das auf die Deutschen durch das Prisma ihrer unverständlichen Sprache schaute, sie zugleich als „nicht unser, geheimnisvoll, geizig, dumm oder lächerlich, auf jeden Fall aber fremd“ (Bystroń 1935/1995: 170) bezeichnete und mit ihnen nichts zu tun haben wollte, sahen sowohl der polnische Mittelstand als auch der polnische Adel in den Deutschen einen wichtigen Handelspartner. Trotz der Wahrnehmung des Deutschen als einer "häretischen“ Sprache der niedrigeren Stufe (im Vergleich zu Latein) lernten sie es im 16. und 17. Jahrhundert aus rein pragmatischen Gründen (Cieśla 1974: 45-46, 50). Die praktischen Ziele, denen der polnische Mittelstand und der Adel beim Deutschlernen folgten, hatten keinen positiven Einfluss auf die gegenseitige deutsch-polnische Wahrnehmung.

Die im 17. Jahrhundert von Wacław Potocki formulierte Feststellung - Solange die Welt besteht, wird der Deutsche dem Polen kein Bruder sein - beschreibt auch besonders zutreffend die deutsch-polnischen Beziehungen in den Zeiten der Teilungen Polens, in denen von den sog. normalen nachbarschaftlichen Verhältnissen keine Rede sein konnte. Die eingeleitete Assimilierungspolitik, die zum Ziel hatte, die Bewohner der besetzten Territorien zu germanisieren, verstärke die Abneigung zur deutschen Sprache, die inzwischen zur offiziellen Amts- und Unterrichtssprache erklärt wurde. Während auf den von Preußen und Österreich annektierten Gebieten Deutsch den Status einer Fremdsprache verlor, wurde es auf den von Russland besetzten Territorien weiter im Sinne einer Fremdsprache unterrichtet. ${ }^{7}$ In der zweiten Hälfte des 19. Jahrhunderts verzichtete Österreich nach und nach auf seine Germanisierungsbestrebungen. Preußen dagegen verstärkte seine Germanisierungspolitik. Mit dem von Bismarck eingeleiteten Kulturkampf wurde die polnische Sprache zugunsten der deutschen völlig verdrängt ${ }^{8}$ (vgl. Cieśla 1974: 188-189, aber auch Grucza 2001a: 1532; 2001b: 580-581; Mackiewicz 2015: 5).

\footnotetext{
${ }^{6}$ Szwargot / szwargotać - eine unverständliche Sprache / unverständlich sprechen.

${ }^{7}$ An dieser Stelle sind die Errungenschaften von der Nationalen Bildungskommission (Komisja Edukacji Narodowej, KEN) zu nennen, vorwiegend ihre Bestrebungen zur Verbreitung und Demokratisierung des Fremdsprachenlehrens und -lernens, (es ging vor allem um Deutsch und Französisch) (vgl. Cieśla 1974: 107-119).

${ }^{8}$ Als Hauptzweck des Deutschlehrens und -lernens wurde die gleichzeitige Verwirklichung von kommunikativen sowie allgemeinbildenden und kognitiven Zielen angesehen (vgl. Cieśla 1974: 190-191).
} 
Um die Jahrhundertwende vom 18. zum 19. Jahrhundert wurde Deutsch nicht mehr als die Sprache des westlichen Nachbarn, des wirtschaftlichen Partners und der Aufklärungsautorität, sondern als die eines strengen und brutalen Besatzers und Feindes wahrgenommen. Nach dem Ersten Weltkrieg behielt Deutsch die Position einer Fremdsprache auf den Gebieten der ehemaligen österreichischen und russischen Besatzung bei. Auf den von Preußen annektierten Territorien wurde anfänglich Deutsch diskriminiert und aus Schullehrplänen gestrichen. Nach der Neubestimmung der Rolle des Deutschunterrichts in den ersten Nachkriegsjahren wurde die deutsche Sprache auch in Großpolen wieder unterrichtet (vgl. Cieśla 1974: 245, siehe dazu auch Grucza 2001b: 581; Mackiewicz 2015: 7). Am Anfang der 30er Jahre des 20. Jahrhunderts befand sich Deutsch mit 60 Prozent der Deutschlernenden auf dem ersten Platz unter den in den polnischen Oberschulen unterrichteten Fremdsprachen. Die nachfolgenden Stellen belegten Französisch mit 30 Prozent und Englisch mit 1,5 Prozent der Oberschullerner (Cieśla 1974: 244). ${ }^{9}$ Die große Popularität der deutschen Sprache in Polen wurde in erster Linie durch utilitaristische Gründe bestimmt.

Die Schrecken des Zweiten Weltkrieges führten dazu, dass Deutsch wieder zur Sprache des Feindes wurde. Die Ziele der nationalsozialistischen Polenpolitik waren nicht mehr auf die Germanisierung der Polen, sondern ihre Versklavung und radikale Vernichtung ausgerichtet, was nach Grucza (1997: 27) am deutlichsten Arthur Greiser, der damalige Gauleiter des Reichsgaus Wartheland, zum Ausdruck brachte:

Es gibt zwischen dem Deutschen und dem Polen keine Gemeinschaft. Ein Eindeutschen von Polen ist, abgesehen von zahlenmäßig geringen Ausnahmen, nicht nur unerwünscht, sondern national-sozialistisch falsch. Der polnische Mensch kann und darf nicht germanisiert werden.

So war der Deutschunterricht auf die Mindestanforderungen beschränkt und diente der Vermittlung der Grundkenntnisse, damit sich die Lernenden (Untermenschen) nur verständlich machen konnten und durch zu gute Deutschkenntnisse nicht die deutsche Sprache entwürdigen. Das Deutschlernen basierte nur auf Erlernen von Wortschatz unter Bewahrung der grammatischen Inkorrektheit. Eine solche Bildungspolitik trug zur Entstehung einer vereinfachten, „,kastrierten“" Form der deutschen Sprache bei (vgl. Grucza 1997: 28, 2001b: 582-583; Mackiewicz 2015: 9; Stasiak 2015: 180).

\footnotetext{
${ }^{9}$ Im Vergleich zu dem Jahr 1929 vergrößerte sich im Jahr 1934 die Zahl der Deutschlernenden um 18 000, beim gleichzeitigen Rückgang (um 26 000) der Französischlernenden (Cieśla 1974: 253).
} 
Im Nachkriegspolen gab es keinen Platz mehr für alles, was an den Deutschen erinnern konnte. Beispielsweise verzichtete man auf die Bezeichnung die Deutschen ganz, wodurch sich die Ratlosigkeit des polnischen Volkes dem Schreckensbild der Besatzung gegenüber offenbarte. Die deutsche Sprache, die man direkt nach Kriegsende mit einer Beleidigung assoziierte, galt somit als verbotene Sprache. Auf dem 1. Bildungskongress, der 1945 in Łódź stattfand, wurde sogar die Möglichkeit debattiert, die deutsche Sprache aus den Bildungsprogrammen völlig auszuklammern. Schließlich wurde der Deutschunterricht in den nach dem Zweiten Weltkrieg zu Polen gehörenden ehemaligen deutschen Gebieten verboten, und in den anderen Regionen auf ein Minimum reduziert (vgl. Wolff-Powęska 2005: 71). Aber selbst die Grausamkeiten des Zweiten Weltkrieges legten das Interesse an der deutschen Sprache und Kultur nicht still. Die Einstellung zum Deutschen in den Nachkriegsjahren änderte sich zuerst an polnischen Universitäten, an denen ungefähr fünf Jahre nach dem Kriegsende Lehrstühle für Germanistik eingerichtet wurden. Um diese Zeit wurde auch der Deutschunterricht in vielen polnischen Schulen wieder angeboten. Das neue Regime der Jahre 1950-1956 schränkte jedoch sowohl die Entwicklung der polnischen Germanistik als auch den Fremdsprachenunterricht stark ein (vgl. Grucza 1997, 2001a, 2001b). Erst nach dem „Auftauen“ im Jahr 1956 kamen bessere Zeiten für den Deutschunterricht in Polen. Das Lernen der deutschen Sprache als Muttersprache der DDR-Bürger, also der Verbündeten der Volksrepublik Polen, wurde von den Bildungsbehörden in polnischen Schulen unterstützt.

Die darauffolgenden Jahre brachten schrittweise eine Verbesserung des Deutschlandbildes in Polen mit sich, aber die noch in den 80er Jahren des 20. Jahrhunderts deklarierte Abneigung den Deutschen gegenüber überwog die erklärte Sympathie, wobei man die Unterscheidung zwischen West- und Ostdeutschen berücksichtigen sollte. Mit dem Systemwechsel in Polen und der Vereinigung der beiden deutschen Staaten begann eine neue sehr produktive Ära in Bezug auf die deutsch-polnischen Beziehungen, die durch eine gute Zusammenarbeit auf vielen Ebenen geprägt war. In den 90er Jahren des letzten Jahrhunderts wurden in Polen zahlreiche Programme ins Leben gerufen sowie die Zusammenarbeit mit vielen deutschen Organisationen und Stiftungen aufgenommen, die den gegenseitigen Austausch der polnischen Lernenden, Studierenden und Lehrenden mit deutschen Muttersprachlern ermöglichten. ${ }^{10} \mathrm{Um}$ diese Zeit erfreute sich Deutsch großer Popularität unter polnischen SchülerInnen, wovon die Zahlen der Deutschlernenden zeugen. Dem vom Zentrum für Bildungsentwicklung [ORE 2011: 5 (sic!)] erstellten Bericht ist zu entnehmen, dass sich vom Schuljahr

${ }^{10}$ Es sind z. B. das Goethe-Institut, das Deutsch-Polnische Jugendwerk (DPJW), der Deutsche Akademische Austauschdienst (DAAD), der Katholische Akademische Ausländer-Dienst (KAAD) sowie Robert Bosch-Stiftung zu nennen. 
1991/1992 bis zum Schuljahr 2002/2003 die Zahl der Deutschlernenden beständig vergrößerte. In den Jahren 2000-2003 erlebte Deutsch als obligatorisches Fach in polnischen Schulen seine Blütezeit, denn es wurde von ca. 2295270 Menschen gelernt. Innerhalb von zwölf Jahren stieg die Gesamtzahl der Deutschlernenden um das Zweieinhalbfache. In den darauffolgenden Jahren, beginnend mit dem Schuljahr 2003/2004, nahm die Zahl der Deutschlernenden jedoch ständig ab.

\section{DIE STELLUNG DER DEUTSCHEN SPRACHE IN POLEN - VOM EU-BEITRITT POLENS BIS ZUR GEGENWART}

Die Übernahme der die Sprachpolitik der Europäischen Gemeinschaft regelnden Verordnungen durch Polen trug zur Neubestimmung des Ranges des Deutschen als Unterrichtsfaches in polnischen Schulen bei. Der Mitteilung der Europäischen Kommission vom 1. August 2005 zufolge

setzt sich die Kommission für eine wirklich mehrsprachige europäische Gesellschaft ein, in der sich die Fremdsprachenkenntnisse kontinuierlich verbessern, bis jeder Bürger neben seiner Muttersprache über praktische Kenntnisse in mindestens zwei weiteren Sprachen verfügt (COM(2005)356: 3; siehe dazu auch COM(2018)272).

Mit zwei weiteren Sprachen sind eine internationale Sprache sowie die Sprache des Nachbarn gemeint. Als Folge dieser Empfehlungen kam es zu einer Bestimmung des Unterrichtsverlaufs bezüglich der ersten und zweiten Fremdsprache in allen polnischen Schultypen. Die genauen Richtlinien wurden in den einzelnen Rahmenlehrplänen ${ }^{11}$ formuliert.

Da seit dem Systemwandel in Polen keine Sprache mehr aufgezwungen wird, werden in den Schulen mehrere Fremdsprachen angeboten. Im letzten Jahrzehnt stand außerhalb jeder Diskussion die Idee, statt Englisch eine andere Fremdsprache als erste Fremdsprache in polnischen Schulen einzuführen. Für jeden, d. h. für die Schulleitung, die SchülerInnen und die Eltern, scheint klar zu sein, dass Englisch einfach die Sprache Nummer Eins, die Weltsprache, die Sprache der internationalen Kommunikation ist. So etablierte sich Englisch als erste Fremdsprache in allen Schultypen in Polen. Skowronek (2016: 136) verweist darauf, dass wegen des weltweit hohen Prestiges des Englischen viele Eltern Englisch als Entwicklungsmöglichkeit und Berufschancen für die Zukunft ihrer Kinder sehen. Dazu kommt die Überzeugung davon, dass Englisch im Vergleich zu anderen Fremdsprachen, z. B. zum Deutschen, einfacher ist.

${ }^{11}$ Siehe Rahmenlehrpläne (Podstawy Programowe) aus zwei letzten Dekaden: Dziennik Ustaw Dz.U. 2002, nr 51, poz. 458; Dz.U. 2009, nr 4, poz. 17; Dz.U. 2012, poz. 977; Dz.U. 2017, poz. 356. 
Es ist anzumerken, dass vor dem EU-Beitritt Polens die Rangfolge der Fremdsprachen in polnischen Schulen [gemeint sind Grundschulen (polnische), Gymnasien ${ }^{12}$ und Oberschulen] anders als heutzutage aussah. Laut Key Data on Teaching Languages at School in Europa 2005 lernten im Jahr 2005 in den polnischen Grundschulen 20,4 Prozent der SchülerInnen Deutsch als obligatorisches Fach, Englisch dagegen nur 9,2 Prozent. In den Gymnasien dominierte Englisch mit 72,6 Prozent der Lernenden. Deutsch lernten 43,2 Prozent der Gymnasiasten. Ähnliche Tendenz war auch in den Oberschulen festzustellen, in denen Englisch von 90,6 Prozent, Deutsch dagegen von 49,3 Prozent der Oberschullerner gelernt wurde (vgl. Komorowska 2007: 26-28). Während sich in dem Zeitraum 2005-2017 die Zahl der polnischen Deutschlernenden in den Grundschulen und in den Oberschulen verringerte, stieg sie in den Gymnasien (vgl. Eurydice 2017: 80).

Englisch als lingua franca des 21. Jahrhunderts gewann seit dem Schuljahr 2009/2010 den Status der ersten Fremdsprache, die ab der ersten Klasse der Grundschule bis zur Oberschule auch heutzutage von den meisten Lernenden gelernt wird. Dem Eurydice-Bericht (2017: 72-73) zufolge lernten im Jahr 2017 über 90 Prozent der polnischen SchülerInnen aller Schultypen Englisch (vgl. dazu auch Auswärtiges Amt 2015). Zu Beginn des Schuljahres 2017/2018 wurde die erste Fremdsprache - meist Englisch - auch im Kindergarten eingeführt. Die zweite Fremdsprache als ein obligatorisches Fach am Gymnasium wird erst seit dem Schuljahr 2009/2010 unterrichtet. Die im Jahr 2017 eingeleitete neue Reform des Schulwesens in Polen, nach der das dreijährige Gymnasium aufgelöst und die Grundschule um zwei Jahre verlängert wird, führt die Pflicht zur zweiten Fremdsprache ab der siebten Klasse ein. In vielen polnischen Grundschulen wurde und wird die zweite Fremdsprache als ein zusätzliches Fach in den Klassen 4-6, je nach den finanziellen Möglichkeiten der Schule, meistens ein bis maximal zwei Mal pro Woche unterrichtet. Nach wie vor ist das Lernen der zweiten Fremdsprache in den Oberschulen obligatorisch.

Da die erste Fremdsprache vom Beginn der Grundschule bis zum Ende der Oberschule ein Pflichtfach ist, wird ihre Fortsetzung auf den einzelnen Bildungsstufen, den in Podstawa Programowa (vgl. Anmerkung 11) formulierten Verordnungen gemäß, garantiert. Der Rahmenlehrplan bestimmt genau das angestrebte Sprachniveau der SchülerInnen nach den jeweiligen Bildungsetappen in Bezug auf den Gemeinsamen Referenzrahmen für Sprachen (Europarat 2001). Während die Feststellung des von den SchülerInnen erreichten Sprachniveaus im Falle der ersten Fremdsprache unproblematisch verläuft, funktioniert sie bei der zwei-

${ }^{12}$ Gymnasium - ein heutzutage nicht mehr bestehender Schultyp, vor 2019 eine drei Jahre lang dauernde Mittelschule, die dem deutschen Sekundarbereich I entsprach. 
ten Fremdsprache in vielen polnischen Schulen kaum wegen der scheinbaren Fortsetzung dieser Sprache auf den nachfolgenden Bildungsstufen. Dies stellt die Verwirklichung der von der Europäischen Kommission verfassten Idee der mehrsprachigen europäischen Gesellschaft in Frage. Auf allen Bildungsstufen, d. h. auf der II., III. und IV., lernen viele SchülerInnen Deutsch ab dem Sprachniveau A1 bzw. A1+. Nicht selten werden in derselben Klasse SchülerInnen mit keinen Deutschkenntnissen und solche zusammen unterrichtet, die seit ein paar Jahren Kontakt zur deutschen Sprache in der Schule haben oder sogar auf den vorherigen Bildungsetappen an verschiedenen Wettbewerben mit Erfolg teilgenommen haben. Resümierend lässt sich sagen, dass die Sprachbildung und Sprachförderung in Polen, besonders bezüglich der Einführung und Fortsetzung der zweiten Fremdsprache, auf vage formulierten und unlogischen Richtlinien basiert. Besorgniserregend ist aber die Tatsache, dass die Autoren jedes sog. neuen Rahmenlehrplans, der die Sprachbildung in Polen eigentlich verbessern sollte, die alten Fehler, statt zu korrigieren, duplizieren. ${ }^{13}$

Podstawa Programowa (siehe Anmerkung 11) zufolge soll die Wahl der zweiten Fremdsprache den Lernenden frei gelassen werden, was in vielen polnischen Schulen seit Jahren nicht der Fall ist. Die "Wahl“ der zweiten Fremdsprache war und ist mehr oder weniger aufgedrängt - in der Grundschule, bis zum Jahr 2017 am Gymnasium fast immer, in der Oberschule nicht immer, aber sehr häufig (dazu siehe auch Mackiewicz 2013: 211). In der Grundschule und am Gymnasium wird / wurde je nach dem angestellten Fremdsprachenlehrer entsprechend Deutsch, Französisch, Russisch, Spanisch bzw. eine andere Fremdsprache gelehrt. Es muss betont werden, dass die Entscheidung darüber, welche Fremdsprache neben Englisch unterrichtet wird, ausschließlich die Schulleitung trifft, oft ohne dies vorher mit den SchülerInnen und ihren Eltern abgesprochen zu haben. In der Oberschule, in der logischerweise die in der Grundschule / am Gymnasium schon gelernte zweite Fremdsprache fortgesetzt werden soll, stehen die Oberschullerner wieder vor der Wahl. In der Tat besteht immerhin zwischen dem Angebot an Fremdsprachen und dem Profil der Klasse ein enger Zusammenhang. Als zweite Fremdsprache wird sowohl in der Grund-als auch in der Oberschule meistens Deutsch gelernt. Manche AutorInnen, die den Stellenwert der deutschen Sprache in Polen fokussieren, berichten, dass Deutsch die am zweithäufigsten gewählte Fremdsprache oder die zweitbeliebteste Fremd-

${ }^{13}$ Von den Glottodidaktikern wurden auf den Seiten ihrer zahlreichen wissenschaftlichen Publikationen nicht nur die Unzulänglichkeiten und Schwächen der einzelnen Rahmenlehrpläne in Hinsicht auf die Realisierung der Mehrsprachigkeit in Polen aufgelistet und kritisiert, sondern auch ganz konkrete Korrekturschritte vorgeschlagen. Siehe dazu z. B. Pfeiffer (2001), Komorowska (2007), Tomaszewska (2009), Wojciechowska (2010), Mihułka (2012, 2016a), Kucharczyk (2014), Abramczyk (2015). 
sprache in Polen ist. ${ }^{14}$ Einerseits scheinen diese Entscheidungen berechtigt zu sein, weil keine andere, natürlich außer Englisch, Fremdsprache von so vielen polnischen SchülerInnen gelernt wird. Andererseits wenn man bedenkt, dass viele Lernende Deutsch gar nicht lernen wollen, sondern müssen, weil keine andere Fremdsprache zur Wahl gestellt wird, bekommen die Bezeichnungen beliebt und gewählt eine ganz andere Bedeutung (siehe dazu auch die emotionelle Färbung dem Deutschen gegenüber in dem historischen Rückblick).

Bezüglich der Zahl der Deutschlernenden in allen polnischen Schultypen außer Hoch- und Sprachschulen sowie Privatunterricht ist seit dem Jahr 2004 eine sinkende Tendenz festzustellen. Dem Report des Zentrums für Bildungsentwicklung (ORE 2011) und den Angaben des Systems für Bildungsinformationen (System Informacji Oświatowej, 2018) zufolge verringerte sich innerhalb der letzten 14 Jahre die Zahl der Deutschlernenden um 821519 Personen, also um ca. 20 Prozent. Während im Schuljahr 2004/ 2005 Deutsch von 2712240 SchülerInnen (davon 2135290 als ein obligatorisches und 576950 als fakultatives Fach) d. h. von 43,4 Prozent gelernt wurde, waren es im Jahr 2018 nur 1890721 Lernende (davon waren 1596737 Personen verpflichtet Deutsch zu lernen und 293984 lernten Deutsch als eine zusätzliche Fremdsprache), d. h. 22,37 Prozent. Wenn man die in Polen gelernten Fremdsprachen berücksichtigt, befindet sich Deutsch trotz der schwierigen Umstände weiterhin auf einem stabilen zweiten Platz.

Laut den Angaben des Systems für Bildungsinformationen (System Informacji Oświatowej, 2018) lernten in den polnischen Bildungseinrichtungen 71,23 Prozent der SchülerInnen Englisch, 22,37 Deutsch, 2,42 Russisch und 1,42 Französisch, was natürlich auch die Wahl der Fremdsprache durch die SchülerInnen, in der die Abschlussprüfung am Ende der Grundschule, des Gymnasiums (bis 2019) und die Reifeprüfung abgelegt wird, stark beeinflusste. Laut den von der Zentralen Prüfungskommission (Centralna Komisja Egzaminacyjna) veröffentlichten Ergebnissen ist die Zahl der Gymnasiasten, die sich während der abschließenden Prüfung in der Fremdsprache für Deutsch entschieden, innerhalb der letzten 10 Jahre um 57297 Personen zurückgegangen. Während im Jahr 2009 noch 94451 Lernende (20,91 Prozent) eine Prüfung in Deutsch ablegten, wählten im Jahr 2019 nur 37154 Gymnasiasten (8,9 Prozent) Deutsch als Prüfungsfach. ${ }^{15}$ Bei der Achtklässler-Prüfung, die das erste Mal im Jahr 2019 stattfand,

\footnotetext{
${ }^{14}$ Siehe u. a. Földes (2016), Łada (2017).

${ }^{15}$ Die Anzahl der Gymnasiasten, die beim Schulabschluss die Prüfung in Deutsch ablegten: 2009 - 94 451; 2010 - 82 696; 2011 - 71 405; 2012 - 93 363; 2013 - 79 616; 2014 - 72 626; 2015 - 54 249; 2016 - 48 467; 2017 - 48 651; 2018 - 43 278; 2019 - 37 154. Zum Vergleich entschieden sich im Jahr 2019591278 Gymnasiasten, also 89,1 Prozent, für Englisch als Prüfungsfach. Es ist dabei zu bemerken, dass seit 2012 bei der Prüfung in Deutsch und Englisch die Einteilung in das Grund- und erweiterte Niveau beibehalten wird. Die obigen Daten umfassen alle Gymnasiasten, die Deutsch /
} 
entschlossen sich für Deutsch nur 15815 Grundschullerner, die 4,4 Prozent aller Prüflinge ausmachten. ${ }^{16}$ Eine ähnliche, ebenfalls sinkende Tendenz, ist im Falle der polnischen Oberschulen bemerkbar. Im Jahr 2005 belief sich die Zahl der Reifeprüflinge, die sich für die Abiturprüfung in Deutsch entschieden, auf 53481 Personen (16,7 Prozent). In den kommenden Jahren verringerte sich ihre Zahl. Nach zehn Jahren (im Jahr 2015) legten Deutsch bei der Reifeprüfung nur 18339 Abiturienten (6,65 Prozent) ab. Die Zahl blieb in den darauffolgenden Jahren konstant, so dass sich im Jahr 201917256 Oberschullerner (6,98 Prozent) einem Abitur in Deutsch unterzogen haben. ${ }^{17}$

An den polnischen Hochschulen lernten im Jahr 2015 gemäß den Angaben des Auswärtigen Amtes (2015) 96555 Studierende Deutsch, was 6 Prozent aller Studierenden ausmachte. Zum Vergleich waren es im Jahr 1996 zwischen 300 000-400 000 Studierende, also ca. ein Drittel der Immatrikulierten (vgl. Grucza 2001a: 1535). ${ }^{18}$

Nach dieser Betrachtung des Ranges des Deutschen an polnischen Schulen in den letzten zwei Dekaden soll nun die Entwicklung des Fremdsprachenerwerbs in Polen untersucht sowie die vorgenommenen Änderungen ausgewertet werden. Als Grundlage der weiteren Ausführungen werden die am Anfang des 21. Jahrhunderts von Pfeiffer (2001: 209) formulierten Prinzipien für die Weiterentwicklung der Schulsprachenpolitik in Polen übernommen, die schon damals als bahnbrechend und weitreichend anerkannt wurden. Die Gegenüberstellung der von Pfeiffer (2001) vor 20 Jahren empfohlenen Leitsätze der aktuellen Schulsprachenpolitik lässt feststellen, ob diese Grundsätze überhaupt und wenn ja, in welchem Umfang in der Schulpraxis umgesetzt wurden. Anhand der in diesem Abschnitt präsentierten Erwägungen kann konstatiert werden, dass die völli-

Englisch abgelegt haben, ohne die erwähnten Niveaus zu unterscheiden. https://cke.gov.pl/images/_EGZAMIN_GIMNAZJALNY/Informacje_o_wynikach/ [Zugriff am: 14.10.2019].

${ }^{16}$ Zum Vergleich wählten 345316 Achtklässler (95,1 Prozent) Englisch während der Abschlussprüfung am Ende der Grundschule. https://cke.gov.pl/egzamin-osmoklasisty/wyniki/ sprawozdanie-z-egzaminu-osmoklasisty-w-2019-r/ [Zugriff am: 14.10.2019].

${ }^{17}$ Die Anzahl der Oberschullerner, die das Abitur in Deutsch ablegten: 2009 - 62 383; 2010 59 195; 2011 - 52 625; 2012 - 50 593; 2013 - 44 238; 2014 - 38 003; 2015 - 18 339; 2016 - 27 427; 2017 - 25 028; 2018 - 20 518; 2019 - 17 256. Englisch als Abiturfach wählten im Jahr 2019 dagegen 381821 Abiturienten. Die vorgeführten Daten umfassen alle Abiturienten, die die Abiturprüfung in Deutsch / Englisch schrieben, ohne dabei auf die Einteilung in das Grund- und erweiterte Niveau einzugehen. https://cke.gov.pl/egzamin-maturalny/egzamin-w-nowej-formule/wyniki/ [Zugriff am: 14.10.2019].

${ }^{18}$ Aus Platzgründen wird im vorliegenden Beitrag auf den Status der polnischen Germanistik nicht eingegangen. Über solche Aspekte, wie: die Geschichte der polnischen Germanistik, ihren institutionellen Zustand, den Vergleich der Zahl der Germanistikstudenten in den einzelnen Jahrzehnten sowie ihre Deutschkenntnisse schreiben u. a. Cieśla (1974), F. Grucza (2001a, 2001b, 2010), Lewicki (2015), Szczęk und Kałasznik (2016). 
ge Umsetzung nur in Bezug auf vier Prinzipien zu verzeichnen ist, und zwar auf: die Ermöglichung des Erlernens von zwei Fremdsprachen an öffentlichen Schulen; die Verringerung des Alters, in dem man beginnt, eine Fremdsprache zu lernen; obligatorisches Lernen von Englisch als zweite Fremdsprache, falls es nicht als erste Fremdsprache gewählt wurde; die Kenntnis mindestens einer Fremdsprache auf dem Niveau der elementaren Kommunikationsfähigkeit. Da diese Fragen am Anfang dieses Abschnitts detailliert besprochen wurden, soll an dieser Stelle nicht mehr drauf eingegangen werden.

Innerhalb der letzten zwanzig Jahre ist es jedoch nicht gelungen, die Wahlmöglichkeit der ersten Fremdsprache durch die SchülerInnen aufrechtzuerhalten, obwohl vor dem EU-Beitritt Polens und ein paar Jahre danach die Wahl der ersten Fremdsprache den Lernenden überlassen wurde. Englisch etablierte sich „offiziell“ im letzten Jahrzehnt als erste Fremdsprache in allen Schultypen in Polen. Von der Wahl einer ersten Fremdsprache ist also keine Rede mehr. Nur teilweise wird seit dem Anfang des 21. Jahrhunderts in den polnischen Schulen das von Pfeiffer (2001: 209) vorgeschlagene Prinzip realisiert, das eine Verbesserung der Sprachvermittlungsbedingungen zum Ziel hat. Die für das Fremdsprachenlehren und lernen bestimmten Klassenräume sind auf jeden Fall besser als zuvor ausgestattet, aber das Postulat die Fremdsprachen in kleinen Gruppen zu unterrichten, wird nicht in allen Schulen (vorwiegend aus ökonomischen Gründen) praktiziert. Das betrifft vor allem die zweite Fremdsprache (Deutsch), die seltener (im Vergleich zu Englisch) von den Lernenden als Prüfungsfach gewählt wird. Seit über zwei Jahrzehnten ist das polnische Bildungssystem jedoch nicht in der Lage, die Fortsetzung des Lernens aller gewählten Fremdsprache(n), vor allem der zweiten, auf den weiterführenden Bildungsstufen zu garantieren. So wird die zweite Fremdsprache (Deutsch) nach wie vor in der Grundschule (bis zum Jahr 2019 am Gymnasium) und in der Oberschule als Fach für Anfänger angeboten. Betrachten wir also den aktuellen Rang der zweiten Fremdsprache (des Deutschen) in der Schulsprachenpolitik in Polen, können die Veränderungen der letzten zwanzig Jahre nicht als eindeutig vorteilhaft betrachtet werden.

\section{ZWISCHENBILANZ}

Die sich in den letzten zwei Dekaden verringerte Zahl der Deutschlernenden wird in erster Linie mit dem Rückgang der Geburtenraten in Polen begründet. Aber das niedrigere Interesse an der deutschen Sprache ist wahrscheinlich nicht nur mit den demografischen Veränderungen zu erklären, sondern auch mit der sinkenden Motivation zum Deutschlernen im Allgemeinen. Dies bestätigen auch die Aussagen der Germanistikstudierenden, die nach dem Abschluss des 
Praktikums in den Schulen formuliert wurden. ${ }^{19}$ Ihnen zufolge ist ungefähr ein Drittel der SchülerInnen zum Deutschlernen motiviert, wobei auf den Unterschied zwischen den stärker zum Lernen dieser Sprache motivierten Grundschullernern und den älteren SchülerInnen (Gymnasiasten und Oberschullernern) hingewiesen wurde (vgl. Mihułka 2016a: 75-76). Im Weiteren werden Gründe der verhältnismäßig geringen Motivation der polnischen SchülerInnen zum Lernen der deutschen Sprache genannt und kurz behandelt. Sie lassen sich in zwei Hauptkategorien einteilen und zwar in: systembedingte Fragen und Einstellung zur deutschen Sprache.

\subsection{Systembedingte Fragen}

In diesem Abschnitt werden Probleme besprochen, deren Lösung nur auf der Regierungsebene liegt.

1. Die scheinbare Fortsetzung der zweiten Fremdsprache in den weiterführenden Bildungsstufen in polnischen Schulen führt auf jeden Fall zur Senkung der Motivation bei den Deutschlernenden. Damit man von einer wirklichen Fortsetzung der zweiten Fremdsprache sprechen könnte, reicht es nur aus, eine obligatorische für alle SchülerInnen vorgesehene Prüfung in der zweiten Fremdsprache am Ende der Grundschule einzuführen. Die Ergebnisse dieser Prüfung würden einerseits teilweise die auf dem Zeugnis stehende Deutschnote beeinflussen, andererseits könnten sie als das wichtigste Kriterium bei der Zuordnung der Lernenden zu entsprechenden Gruppen, je nach Sprachniveau, in der Oberschule angesehen werden (siehe dazu Mihułka 2012: 341; 2016a: 77-78).

2. Die zu niedrige Stundenzahl, die für den Unterricht in der zweiten Fremdsprache in allen Schultypen vorgesehen ist. In der siebten und achten Klasse der Grundschule haben die Lernenden Deutsch nur zwei Mal pro Woche. In der Oberschule, im Grundniveau, ist die Stundenzahl gleich. Dabei achtet man in den Schulen nicht immer darauf, dass die Deutschstunden innerhalb der Woche sinnvoll verteilt sind. Es kommt vor, dass die SchülerInnen Deutsch an den nachfolgenden Tagen, manchmal sogar an demselben Tag lernen (vgl. dazu Abramczyk 2015: 127-128).

${ }^{19}$ Im Abschnitt 4 beruft man sich auf die Ergebnisse der Umfrage, die im Jahr 2016 unter den Germanistikstudierenden der Universität Rzeszów direkt nach dem Abschluss des Praktikums in den Grundschulen, Gymnasien und Oberschulen durchgeführt wurde. Es wurde $\mathrm{u}$ a. nach der Einstellung der polnischen Deutschlernenden (unterschiedlichen Alters) dem Deutschen gegenüber sowie ihrer Motivation zum Deutschlernen gefragt (siehe Mihułka 2016a). 
3. Langweilige Lehrmaterialien für Deutsch als Fremdsprache, die mit den die Sprachbildung in Polen regelnden Dokumenten nur teilweise oder gar nicht im Einklang stehen. In diesem Punkt werden zwei Probleme angesprochen, und zwar: 1. Viele vom Ministerium für Schulwesen genehmigte Lehrmaterialien für Deutsch als Fremdsprache entsprechen nur in Teilbereichen den Richtlinien der in einzelnen Zeiträumen geltenden Rahmenlehrplänen (vgl. Anmerkung 11) sowie den Verordnungen und Empfehlungen des Gemeinsamen Europäischen Refernzrahmen für Sprachen (Europarat 2001). 2. Viele auf dem Buchmarkt erhältliche Lehrmaterialien für Deutsch als Fremdsprache werden von den Lernenden als langweilig, monoton, oberflächlich oder schablonenhaft beurteilt. Der Gesichtspunkt der SchülerInnen wird in manchen Fällen auch von den sie unterrichtenden Deutschlehrenden geteilt. Das Problem liegt aber darin, dass die an öffentlichen Schulen arbeitenden Fremdsprachenlehrenden nicht immer die Möglichkeit haben, über die Wahl der Lehrmaterialien selbst zu entscheiden (siehe dazu Abramczyk 2015: 128-129).

4. Keine Beachtung der Kenntnis der von den Lernenden früher gelernten (Fremd)Sprachen. Es sollten entsprechende Lehrprogramme, Lehrmaterialien konzipiert werden, die die Kenntnis des Englischen und Polnischen beim Lernen des Deutschen berücksichtigen. Das gleiche bezieht sich auch auf die Einführung der verbindlichen Änderungen in den Programmen zur Ausbildung von Deutschlehrern (vgl. dazu u. a. Mihułka 2016a: 78-79; Kucharczyk 2014: 252).

5. Die vermeintliche Wahl der zweiten Fremdsprache durch die SchülerInnen, vorwiegend in der Grundschule (am Gymnasium) und teilweise in der Oberschule. Einerseits scheint die Auferlegung des Deutschen, also der Sprache unseres in der Europäischen Union einflussreichen und aus der Sicht der Weltwirtschaft bedeutsamen Nachbarn als zweiten Fremdsprache in der Grundschule (vor der neuen Schulreform auch am Gymnasium) und teils in der Oberschule, gerechtfertigt zu sein. Andererseits weiß man aus der Geschichte, dass die Lernenden eine aufgedrängte Sprache nicht immer gern lernen und positiv wahrnehmen. Als Beispiel kann die negative Einstellung vieler polnischer SchülerInnen dem Russischen gegenüber vor und nach dem Systemwandel in Polen angegeben werden.

\subsection{Einstellung der deutschen Sprache gegenüber}

Laut den Aussagen der angehenden Deutschlehrenden ist die erhebliche Mehrheit der Lernenden (70 Prozent) dem Deutschen gegenüber negativ ein- 
gestellt. Je älter die SchülerInnen sind, desto negativer ist ihr Verhältnis zu dieser Sprache (Mihułka 2016a: 72). Die negative Einstellung der Lerner zum Deutschen hängt von einer Reihe von Gründen $a b$, von denen nur die von den Polen am häufigsten genannten ${ }^{20} \mathrm{im}$ Folgenden angeführt und zur Diskussion gestellt werden.

1. Der Klang der deutschen Sprache. Die deutsche Sprache wird nach wie vor als eine "harte", "schwere" und "schreckliche“ Sprache bezeichnet.

2. Der Schwierigkeitsgrad des Deutschen. Als Grund der Feststellung ich mag Deutsch nicht - wird oft die Überzeugung von der besonderen Kompliziertheit und Schwierigkeit der deutschen Sprache angegeben.

3. Die Bedeutung und Nützlichkeit des Deutschen in der Welt. Viele (junge) Polen gehen davon aus, dass die Englischkenntnisse ausreichend sind, um sich beruflich und privat überall zurechtzufinden (auch in den deutschsprachigen Ländern). So sind viele von der geringen Bedeutung der deutschen Sprache in der Welt überzeugt, und deswegen sehen sie keinen Sinn darin, Zeit und Energie dem Erlernen dieser Sprache zu widmen.

4. Die Qualität des Deutschunterrichts gilt als eins der Merkmale, die die Motivation der SchülerInnen zum Deutschlernen stärken bzw. abschwächen können. Das Wort Qualität bezieht sich sowohl auf das Lehrpersonal, seine Ausbildung (Fach- und Sprachkompetenzen) sowie seine Persönlichkeit und soft skills, als auch auf die Planung und Durchführung des Unterrichts mit der Berücksichtigung der eingesetzten Lehrmaterialien. Da die SchülerInnen beispielweise den Deutschunterricht mit einem ganz konkreten Lehrkörper verbinden, kommt es nicht selten vor, dass sie ihre Einstellung dem Deutschlehrköper gegenüber auch auf das Fach und die deutsche Sprache übertragen..$^{21}$

5. Die Einstellung den Deutschen gegenüber. Das nicht immer positive Verhältnis der Polen zur deutschen Sprache resultiert auch aus der nicht immer positiven Einstellung ihren Trägern gegenüber. Obwohl innerhalb der letzten zwanzig Jahre der Akzeptanzgrad der Deutschen durch Polen und die ihnen erklärte Sympathie erheblich stiegen (vgl. u a. Dolińska \& Fałkowski 2003; Fronczyk \& Łada 2009; Mihułka 2012: 293-303; Łada

${ }^{20}$ Die kurze Besprechung der einzelnen Punkte knüpft an die Untersuchungsergebnisse (Mihułka 2012, 2016a; Gajewska-Daszkiewicz 2012; Mackiewicz 2013; Łada 2018b) an, die vorwiegend unter den Deutschlernenden und den (zukünftigen) Deutschlehrenden, aber auch durchschnittlichen Polen (derer Kontakt zu der deutschen Sprache nicht näher zu bestimmen ist) durchgeführt wurden.

${ }^{21}$ Mehr über das Bild eines guten Fremdsprachenlehrers in den Augen der Lernenden aller Schultypen in Polen schreiben Mihułka und Chojnacka-Gärtner (2019). 
2018a), können sich einige Polen von der stereotypenhaften Wahrnehmung des westlichen Nachbarn und seiner zu einseitigen Beurteilung nicht befreien, was das Verhältnis zur deutschen Sprache direkt beeinflusst.

\section{SCHLUSSWORT}

Die in den vorherigen Abschnitten besprochene Stellung der deutschen Sprache in Polen, besonders im 21. Jahrhundert, sollte einen Überblick bieten, der sich grundsätzlich auf den Befunden empirischer Untersuchungen sowie Meldungen, Mitteilungen und Berichten der den Verlauf der Sprachbildung in Polen überwachenden Institutionen stützt. Die vorgestellten Überlegungen sind frei von emotionalen Formulierungen und basieren hauptsächlich auf Fakten, die nur an einigen Stellen mit einem Kommentar versehen sind. Es ist aber nicht einfach einen Text ohne Emotionen zu schreiben, wenn einem, $u$. a. aufgrund der eigenen beruflichen Tätigkeit, der Stellenwert der deutschen Sprache in Polen, die Einstellung der (Deutsch)Lernenden dem Deutschen und den Deutschen selbst gegenüber nicht gleichgültig sind. Daher erlaube ich mir an dieser Stelle, etwas vom wissenschaftlichen Schreibstil abzuweichen und im Folgenden das Schlusswort aus der Perspektive eines Deutschlehrenden zu formulieren.

In erster Linie möchte ich auf die Probleme eingehen, auf die wir als Deutschlehrer abgesehen von dem Schultyp, in dem wir tätig sind, nur begrenzten Einfluss haben. Die Fragen der Gestaltung der polnischen Schulsprachenpolitik sind für uns unerreichbar, denn wir können weder über Fragen der Fortsetzung des Deutschen in den weiterführenden Bildungsstufen mitentscheiden noch haben wir Einfluss auf die geplante Stundenzahl für diese Sprache an einzelnen Schultypen sowie auf die Form der DaF-Lehrmaterialien. Das bedeutet aber nicht, dass wir alles in Kauf nehmen müssen, was uns angeboten wird. Wir sind dazu verpflichtet, auf die Unzulänglichkeiten und Lücken des Rahmenlehrplans hinzuweisen sowie gegen die derzeitige Form der Sprachbildung und Sprachförderung in Bezug auf die zweite Fremdsprache Einwände zu erheben. Die systembedingten Fragen scheinen außerhalb unserer Möglichkeiten zu liegen, aber das heißt nicht, dass wir gar nichts machen können, um die SchülerInnen aller Altersgruppen zum Deutschlernen zu motivieren und ihnen zu zeigen, dass das Deutschlandbild vielfältig sein kann sowie dass kriegsbezogene Assoziationen die Werte des Landes und die positiven Eigenschaften seiner Bewohner nicht überschatten müssen. Wir brauchen dabei keine spektakulären Schritte zu unternehmen, um das Wissen der SchülerInnen über unseren westlichen Nachbarn zu erweitern und sie für das Anderssein zu sensibilisieren. Die Ergebnisse der 
Forschungsprojekte (z. B. von Adamczak-Krysztofowicz 2003; Kubiczek 2016; Chudak 2016) bestätigen, dass der Einbezug authentischer Materialien (Texte, Filme) in den Deutschunterricht und die zielgerichtete Arbeit mit ihnen nicht nur die Vorstellung der Deutschlernenden über Deutschland und die Deutschen positiv verändern, sondern sie auch zum Deutschlernen anspornen können.

Das Kennenlernen der deutschen Kultur hat seinen Anfang im Ausgangsland (in Polen), kann aber im Zielsprachenland (in Deutschland) fortgesetzt werden. Deswegen ist es empfehlenswert, den Lernenden den direkten Kontakt zu der Zielsprachenkultur und ihren Trägern zu ermöglichen. Die Teilnahme an verschiedenen deutsch-polnischen Austauschprogrammen kann durch das persönliche Erleben der fremden Kultur(en) und das Kennenlernen ihrer Vertreter vor Ort sowohl Neugier auf die Welt und Aufgeschlossenheit dieser Kultur(en) gegenüber entfalten als auch die stereotypenhafte Auffassung von Deutschland und Deutschen bei den Austauschteilnehmern relativieren sowie dem Deutschlernen selbst einen neuen Sinn verleihen (vgl. dazu Adamczak-Krysztofowicz, Schmidt-Bernhardt, Stork \& Rybszleger 2011; Gorąca-Sawczyk \& Jourdy 2017; Bochnia 2018). Ansonsten sollen wir in jeder Deutschstunde die SchülerInnen daran erinnern und darauf aufmerksam machen, dass Deutsch, wie Ammon (2015) hervorhebt, eine Weltsprache ist, die es sich zu lernen lohnt, auch wenn es nur aus wirtschaftlichen und pragmatischen Gründen geschieht. Den Lernenden sollten wir jeder Zeit bewusst machen, dass man an der Sprache unseres wirtschaftlich sehr gut entwickelten Nachbarn nicht einfach gleichgültig vorbeigehen darf.

\section{LITERATURVERZEICHNIS}

Abramczyk, A. (2015). Co „gryzie” nauczycieli języków obcych? Języki Obce w Szkole, 4, 126-130.

Adamczak-Krysztofowicz, S. (2003). Texte als Grundlage der Kommunikation zwischen Kulturen. Eine Studie zur Landes- und Kulturkundevermittlung im DaF-Studium in Polen. Hamburg: Dr. Kovač.

Adamczak-Krysztofowicz, S. / Schmidt-Bernhardt, A. / Stork, A. / Rybszleger, P. (2011). Deutsch-polnische Entdeckungen. Projektarbeit zur Förderung interkultureller Kompetenz in Schule und Studium. Theoretische Grundlagen und praktische Lernmaterialien. Marburg: Tectum.

Ammon, U. (2015). Die Stellung der deutschen Sprache in der Welt. Berlin / München / Boston: de Gruyter.

Auswärtiges Amt, (2015). Deutsch als Fremdsprache weltweit. Datenerhebung 2015. Berlin. http:/ / www. auswaertiges-amt.de/cae/servlet/contentblob/364458/publicationFile/204449/PublStatistik. pdf, [Zugriff am: 15.04.2019].

Bochnia, E. (2018). „Rozwijanie kompetencji interkulturowej poprzez wymiany młodzieżowe w kształceniu zawodowym". Unveröffentlichte Dissertation. Poznań: UAM.

Brzozowska, D. (2008). Polski dowcip etniczny. Stereotyp a tożsamość. Opole: Wydawnictwo Uniwersytetu Opolskiego.

Bystroń, J. S. (1935/1995). Megalomania narodowa. Warszawa: Książka i Wiedza. 
Centralna Komisja Egzaminacyjna,

https://cke.gov.pl/egzamin-maturalny/egzamin-w-nowej-formule/wyniki/ https://cke.gov.pl/egzamin-maturalny/egzamin-w-starej-formule/wyniki/

https://cke.gov.pl/egzamin-osmoklasisty/wyniki/sprawozdanie-z-egzaminu-osmoklasistyw-2019-r/

https://cke.gov.pl/images/_EGZAMIN_GIMNAZJALNY/Informacje_o_wynikach/ [Zugfiff am: 14.10.2019].

Chudak, S. (2016). Ogród działkowy jako polsko-niemieckie miejsce pamięci? Film jako bodziec do refleksji na temat tego, co wspólne, oddzielne, paralelne. In: A. Jaroszewska / B. Karpeta-Peć / M. Smuk / J. Sobańska / J. Sujecka-Zając (Hrsg.). Wielojęzyczność i międzykulturowość na lekcji języka obcego. Między teorią a praktyka nauczania (S. 233-250). Warszawa: Instytut Germanistyki Uniwersytetu Warszawskiego / Instytut Romanistyki Uniwersytetu Warszawskiego.

Cieśla, M. (1974). Dzieje nauki języków obcych w zarysie. Warszawa: Państwowe Wydawnictwo Naukowe.

Dolińska, X. / Fałkowski, M. (2003). Polska - Niemcy. Wzajemny wizerunek. In: L. Kolarska-Bobińska (Hrsg.). Obraz Polski i Polaków w Europie (S. 203- 266). Warszawa: Instytut Spraw Publicznych.

Dziennik Ustaw (2002). Rozporzadzenie MENiS z dnia 26.02.2002 r. w sprawie podstawy programowej wychowania przedszkolnego oraz ksztatcenia ogólnego w poszczególnych typach szkót. Dz.U. 2002, nr 51 poz. 458. http:// prawo.sejm.gov.pl/isap.nsf/DocDetails.xsp?id=WDU20020510458 [Zugriff am: 14.10.2019].

Dziennik Ustaw (2009). Rozporządzenie Ministra Edukacji Narodowej z dnia 23 grudnia 2008 r. $w$ sprawie podstawy programowej wychowania przedszkolnego oraz ksztatcenia ogólnego w poszczególnych typach szkót. Dz.U. 2009, nr 4 poz. 17. http:/ / prawo.sejm.gov.pl/isap.nsf/DocDetails. xsp?id=WDU20090040017 [Zugriff am: 14.10.2019].

Dziennik Ustaw (2012). Rozporzadzenie Ministra Edukacji Narodowej z dnia 27 sierpnia 2012 r. w sprawie podstawy programowej wychowania przedszkolnego oraz kształcenia ogólnego w poszczególnych typach szkót. Dz.U. 2012, poz. 977, ze zm. http://prawo.sejm.gov.pl/isap.nsf/DocDetails. xsp?id=WDU20120000977 [Zugfiff am: 14.10.2019].

Dziennik Ustaw (2017). Rozporzadzenie Ministra Edukacji Narodowej z dnia 14 lutego 2017 r. w sprawie podstawy programowej wychowania przedszkolnego oraz podstawy programowej ksztatcenia ogólnego dla szkoty podstawowej, w tym dla uczniów z niepetnosprawnościa intelektualną w stopniu umiarkowanym lub znacznym, kształcenia ogólnego dla branżowej szkoły I stopnia, kształcenia ogólnego dla szkoły specjalnej przysposabiającej do pracy oraz kształcenia ogólnego dla szkoły policealnej. Dz.U. 2017, poz. 356. http:/ / prawo.sejm.gov.pl/isap.nsf/DocDetails.xsp?id=WDU20170000356 [Zugriff am: 14.10.2019].

Europäische Kommission (2005). Die Mitteilung der Kommission an das Europäische Parlament und den Rat (Europäischer Indikator für Sprachenkompetenz. COM(2005)356. http:/ / www.europarl. europa.eu/RegData/docs_autres_institutions/commission_europeenne/com/2005/0356/ COM_COM(2005)0356_DE.pdf [Zugriff am: 14.10.2019].

Europäische Kommission (2018). Vorschlag für eine Empfehlung des Rates zu einem umfassenden Ansatz für das Lehren und Lernen von Sprachen; Anhang der Empfehlung des Rates zu einem umfassenden Ansatz für das Lehren und Lernen von Sprachen. COM (2018) 272. https:/ / eur-lex.europa.eu/resource.html?uri=cellar:1cc186a3-5dc7-11e8-ab9c 01aa75ed71a1.0003.02/DOC_1\&format=PDF [Zugriff am: 24.10.2019].

Europarat für kulturelle Zusammenarbeit (2001). Gemeinsamer europäischer Referenzrahmen für Sprachen:lernen, lehren, beurteilen. Berlin / München / Wien / Zürich / New York: Langenscheidt.

Eurydice (2017). Key data on teaching languages at school in Europe - 2017 edition (Kluczowe dane o nauczaniu języków w szkołach w Europie - 2017). Luksemburg: Urząd Publikacji Unii Europej- 
skiej. https://eurydice.org.pl/wp-content/uploads/2017/12/KDL_PL_2017.pdf [Zugriff am: 26.10.2019].

Földes, C. (2016). Position und Entwicklungsdynamik des Deutschen als Fremdsprache international - im Blick der angewandten Linguistik. Glottodidactica. An International Journal of Applied Linguistics, 43 (2), 13-38.

Fronczyk, A. / Łada, A. (2009). Niemcy i polityka europejska w oczach Polaków. In: L. Kolarska-Bobińska / A. Łada (Hrsg.). Polska - Niemcy. Wzajemny wizerunek i wizja Europy (S. 127-165). Warszawa: Instytut Spraw Publicznych.

Gajewska-Dyszkiewicz, A. (2012). Opinie polskich gimnazjalistów dotyczące nauki języków obcych. Jezzyki Obce w Szkole, 4, 73-78.

Gorąca-Sawczyk, G. / Jourdy, N. (2017). Schüleraustausch als Weg zur Entwicklung interkultureller Kompetenz - deutsch-französische und deutsch-polnische Austauschprojekte im schulischen Bereich. Colloquia Germanica Stetinensia, 26, 271-285.

Grucza, F. (1997). „Kommt reden wir zusammen, wer redet, ist nicht tot.“ Vom Verhältnis der Polen zur deutschen Sprache. Wirtschaft \& Wissenschaft, 2, K2132F, 27-35.

Grucza, F. (2001a). Deutschunterricht und Germanistikstudium in Polen. In: G. Helbig / L. Götze / G. Henrici / H. J. Krumm (Hrsg.). Deutsch als Fremdsprache. Ein internationales Handbuch. 2. Halbband (S. 1528-1543). Berlin / New York: de Gruyter.

Grucza, F. (2001b). Zur Geschichte und Zukunft des Deutschen in Polen: Die Sicht eines Kaschuben. Studia Niemcoznawcze, 22, 577-592.

Grucza, F. (2010). Deutsch in Polen. In: H. J. Krumm / Ch. Fandrych / B. Hufeisen / C. Riemer (Hrsg.). Deutsch als Fremd- und Zweitsprache. Ein internationales Buch. Bd. 2 (S. 1761-1766). Berlin / New York: de Gruyter.

Janoszczyk, J. (2016). Deutsch - (immer noch?) die zweitpopulärste Fremdsprache in Polen. In: K. Mihułka / M. Sieradzka / R. Budziak (Hrsg.). Die Fremdsprache Deutsch in Polen: Anfünge, Gegenwart, Perspektiven (S. 43-60). Rzeszów: Wydawnictwo Uniwersytetu Rzeszowskiego.

Komorowska, H. (2007). Polska polityka językowa na tle innych krajów Unii Europejskiej. In: H. Komorowska (Hrsg.). Nauczanie języków obcych - Polska a Europa (S. 13-36). Warszawa: Wydawnictwo SWPS Academica.

Kopaliński, W. (2000). Stownik wyrazów obcych i zwrotów obcojezycznych z almanachem. Warszawa: Świat Książki.

Kubiczek, A. (2016). Einblicke der Jugendlichen in die deutsche Realität, Mentalität und Sprache mit Anwendung authentischer Lesetexte im Fremdsprachenunterricht. In: K. Mihułka / P. Bąk / J. Chojnacka-Gärtner (Hrsg.). Interkulturalität in Theorie und Praxis der Glottodidaktik und Translatorik (S. 73-87). Rzeszów: Wydawnictwo Uniwersytetu Rzeszowskiego.

Kucharczyk, R. (2014). Wielojęzyczność w polskiej szkole - sukces czy porażka? Kilka słów o wdrażaniu w życie nowej podstawy programowej. In: J. Sujecka-Zając / A. Jaroszewska / K. Szymankiewicz / J. Sobańska-Jędrych (Hrsg.). Inspiracja, motywacja, sukces. Rola materiałów dydaktycznych i form pracy na lekcji języka obcego (S. 241-255). Warszawa: Instytut Germanistyki i Romanistyki UW.

Lewicki, R. (2015). Dem Germanistikstudium einen neuen Sinn verleihen. Konińskie Studia Językowe, 3 (2), 159-173.

Łada, A. (2017). Floskeln oder Fakten? Zur Situation der deutschen Sprache in Polen. Fakten und Meinungen. Warszawa: Instytut Spraw Publicznych.

Łada, A. (2018a). Polacy i Niemcy. Dzielaca przeszłości, wspólna przyszłość? Wyniki Barometru. Warszawa: Instytut Spraw Publicznych.

Łada, A. (2018b). Trudne partnerstwo. Polacy i Niemcy o kraju sąsiada, wspólnej historii i Europie. Warszawa: Instytut Spraw Publicznych. 
Mackiewicz, M. (2013). Motywacja i nastawienia polskich uczniów do nauki języka niemieckiego w kontekście różnic regionalnych i postrzegania społeczeństwa niemieckiego. Neofilolog, 40/2, 205-217.

Mackiewicz, M. (2015). Język niemiecki w Polsce. In: Interakcje. Leksykon komunikowania polsko-niemieckiego. www.polska-niemcy-interakcje.pl [Zugriff am: 12.04.2019].

Mihułka, K. (2012). Rozwój kompetencji interkulturowej w warunkach szkolnych - mity a szkolna rzeczywistość. Na przykładzie języka niemieckiego jako obcego. Rzeszów: Wydawnictwo Uniwersytetu Rzeszowskiego.

Mihułka, K. (2016a). Der Deutschunterricht in Polen - Bemerkungen der Germanistikstudenten der Universität Rzeszów nach dem Abschluss des pädagogisch-didaktischen Praktikums. In: K. Mihułka / M. Sieradzka / R. Budziak (Hrsg.). Die Fremdsprache Deutsch in Polen: Anfänge, Gegenwart, Perspektiven (S. 61-82). Rzeszów: Wydawnictwo Uniwersytetu Rzeszowskiego.

Mihułka, K. (2016b). Die Entwicklung der interkulturellen Kompetenz im DaF-Unterricht - ein Meilenstein im deutsch-polnischen Dialog. Zum Deutschlandbild der Polen, Polenbild der Deutschen und interkulturellen / deutsch-polnischen Dialog. In: M. Wierzbicka / L. Wille (Hrsg.). Im Wirkungsfeld der kontrastiven und angewandten Linguistik / In the field of contrastive and applied linguistics. Bd. 7 (S. 74-91). Rzeszów: Wydawnictwo Uniwersytetu Rzeszowskiego.

Mihułka, K. / Chojnacka-Gärtner, J. (2019). Auf die Persönlichkeit des Lehrers kommt alles an Forschungsbericht zum Profil eines "guten" Fremdsprachenlehrers in allen Schultypen in Polen. Journal of Languages for Specific Purposes, 6, 167-176.

Ośrodek Rozwoju Edukacji (=ORE). (2011). Nauczanie jezzyka niemieckiego w Polsce. Raport przegladowy 1990-2010 sporzadzony przez ORE na zlecenie MEN. Warszawa: ORE.

Pfeiffer, W. (2001). Nauka języków obcych. Od praktyki do praktyki. Poznań: Wagros.

Skowronek, B. (2016). Germanistische glottodidaktische Forschung in Polen: Retrospektive und Perspektive. In: E. Żebrowska / M. Olpińska-Szkiełko / M. Latkowska (Hrsg.). Zwischen Kontinuität und Modernität. Metawissenschaftliche und wissenschaftliche Erkenntnisse der germanistischen Forschung in Polen (S 128-141). Warszawa: Stowarzyszenie Germanistów Polskich.

Stasiak, H. (2015). Kontakt, Kommunikation, Verständnis. Perspektiven für Deutsch als Kontaktsprache in Europa. Konińskie Studia Jezzykowe, 3 (2), 175-185.

System Informacji Oświatowej (2018). Języki obce wg województw. https:/ / cie.men.gov.pl/sio-stronaglowna/dane-statystyczne/jezyki-obce-dane-statystyczne/ [Zugriff am: 23.10.2019].

Szczęk, J. / Kałasznik, M. (2016). Deutsch als Fremdsprache im universitären Bereich - Diagnose und Perspektiven für die Zukunft. In: K. Mihułka / M. Sieradzka / R. Budziak (Hrsg.). Die Fremdsprache Deutsch in Polen: Anfünge, Gegenwart, Perspektiven (S. 99-116). Rzeszów: Wydawnictwo Uniwersytetu Rzeszowskiego.

Tomaszewska, B. (2009). Lekcja w szkole ponadpodstawowej i ponadgimnazjalnej. In: H. Komorowska (Hrsg.). Skuteczna nauka języka obcego. Struktura i przebieg zajęćjęzykowych (S. 142-158). Warszawa: CODN.

Wojciechowska, B. (2010). Wielojęzyczność w polskim kontekście edukacyjnym. Neofilolog, 35, 63-74. Wolff-Powęska, A. (2005). Co Niemiec, to odmieniec. Polityka, 18 (2502), 70-71.

Received: 29.11.2019; revised: 12.03 .2020

\author{
KRYSTYNA MIHUŁKA \\ Uniwersytet Rzeszowski \\ kmihulka@gmail.com
}

ORCID: 0000-0002-8665-4296 
